\title{
Aprendizaje cooperativo en la producción de textos en inglés en
} estudiantes de secundaria JEC

\section{Cooperative learning in the english production texts in the students of first grade of} secondary JEC "Julio C. Tello".

Jossara Evellyn , Fernández Blanco ${ }^{a}$ evelin.unu@ gmail.com

José Smith, Landeo Cabezudo b Josecabezudo1990@ hotmail.com

Elizabeth, Pacheco Dávila ${ }^{\mathrm{c}}$ elichay_9@hotmail.com

a Universidad Nacional de Ucayali

${ }^{\mathrm{b}}$ Universidad Nacional de Ucayali

${ }^{c}$ Universidad Nacional de Ucayali

Recibido: Enero/ 06/2020 • Aceptado: Abril/02/2020 • Publicado: Abril/30/2020

\section{RESUMEN}

El presente trabajo de investigación tuvo como objetivo determinar cómo influye el Aprendizaje cooperativo en la producción de textos inglés. Es una investigación de tipo experimental y diseño cuasi-experimental. Los resultados en el post test del grupo control, respecto a la influencia del aprendizaje cooperativo en la producción de textos en inglés presentan un $27 \%$ que se encuentra en nivel inicio, el $53 \%$ en proceso y un $20 \%$ en esperado; mientras que en el post test del grupo control un $7 \%$ se encuentra en nivel inicio, el 17 $\%$ en proceso un $50 \%$ en esperado y un $27 \%$ en el nivel destacado. Se concluye que el Aprendizaje cooperativo influye significativamente en el desarrollo de la Producción de textos en el área de inglés en los estudiantes del primer grado de educación secundaria de la institución educativa JEC "Julio C. Tello".

Palabras clave: Aprendizaje cooperativo, producción de textos, estrategia metodológica.
ABSTRACT

This research work aimed to determine how cooperative learning influences the production of English texts. It is an experimental research and quasi-experimental design. The results in the post-test of the control group, regarding the influence of cooperative learning on the production of texts in English, present $27 \%$ that are at the beginning level, $53 \%$ in process and $20 \%$ in expected learning; while in the post-test of the control group $7 \%$ is at the initial level, $17 \%$ is in process, $50 \%$ is expected learning and $27 \%$ is at the outstanding level. It is concluded that cooperative learning significantly influences the development of the production of texts in the English area in the students of the first grade of secondary education of the JEC "Julio C. Tello" school.

Keywords: Cooperative learning, text production, methodological strategy.

${ }^{a}$ Jossara Evellyn Fernández-Blanco, ${ }^{\mathrm{b}}$ José Smith Landeo-Cabezudo y ${ }^{\mathrm{c}}$ Elizabeth Pacheco-Dávila Articulo Protegido por Licencia Creative Commons: BY-NC-ND / Protected by Creative Commons: BY-NC-ND. Sendas es una revista de acceso abierto / Sendas is an Open Access Journal. 


\section{INTRODUCCIÓN}

El presente artículo pretende determinar la influencia del aprendizaje cooperativo en la producción de textos en inglés en estudiantes del $1^{\circ}$ de secundaria JEC "Julio C. Tello". Este interés surge porque en el nivel de secundaria aún persisten profundas falencias en el manejo del inglés a pesar de la inversión en capacitación a los docentes que realizó el estado para revertir esta situación. Al respecto, sobre el dominio de esta lengua extranjera en el Perú, publica ANDINA (2016) lo siguiente:

El Perú se encuentra en el puesto 45 (nivel bajo) en un "ranking" mundial sobre el dominio de idioma inglés que incluye a 72 países del mundo y que busca difundir los beneficios educativos y económicos del aprendizaje de dicho idioma. (párraf. 1)

Tener dominio del idioma inglés en la actualidad es una necesidad por ser una de las lenguas que más se habla en el mundo, ya que nos permite comunicarnos y a su vez estar actualizados en los diferentes campos del saber humano, pero sobre todo escribir en una segunda lengua para exponer nuestras ideas con claridad. A esta realidad hay que sumar el descuido de los educadores en promover los principios, valores y las prácticas de desarrollo social en la enseñanza y el aprendizaje (Unesco, 2014)) para que tengan un exitoso performance en distintas situaciones y contextos.

El aprendizaje es individual, pero también si este aprendizaje no se socializa se torna inútil, ya que la construcción del conocimiento es el resultado de la construcción del individuo y del grupo social (Vigotsky, citado por Duran y Vidal, 2009).

Los estudiantes en general son dinámicos, están en permanente interacción con otros, entonces urge la necesidad de canalizar estas energías en provecho de la producción de textos en inglés y el desarrollo de principios y valores. El método que se ajusta a esta necesidad es el aprendizaje cooperativo.

Sobre esta problemática, existen investigaciones que tienen relación directa con el presente trabajo, como la que plantea De León (2013). Él concluyó que la implementación de la metodología de aprendizaje cooperativo en el curso de inglés permitió un resultado

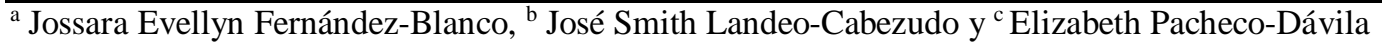
Articulo Protegido por Licencia Creative Commons: BY-NC-ND / Protected by Creative Commons: BY-NC-ND. Sendas es una revista de acceso abierto / Sendas is an Open Access Journal. 
positivo en el grupo experimental, más no significativo en términos estadísticos. Además, se observó un cambio en la conducta social de los estudiantes, quienes manifestaron valores y actitudes de importancia. Mientras que Jara et al (2016) sostiene que el aprendizaje cooperativo mejora el proceso de aprendizaje de la lengua extranjera en los alumnos de primer año, ya que se logró que los alumnos trabajen cooperativamente, se ayuden al interior del grupo y cooperen con otros grupos; situación que propició la mejoría en el aprendizaje. Por otra parte, Gutiérrez (2015) concluyó que la aplicación del programa de las estrategias de aprendizaje cooperativo influye significativamente en la mejora de la producción de textos.

La iniciativa de realizar esta investigación surgió durante la práctica pre profesional, ya que se observó entre los estudiantes de la Institución Educativa JEC “Julio C. Tello”, poco dominio del idioma inglés. A esta realidad se suma que en el contexto regional se observa el poco interés de algunos profesores para realizar acciones pedagógicas de aprendizaje cooperativo que contribuyan con la comunicación asertiva en la lengua inglesa, más aún en la escritura. Es evidente, además, que gran parte de los profesores de inglés prefieren mantener la situación tal como se presenta o se ven limitados en su capacidad de introducir innovaciones pedagógicas para revertirlo debido a su poca competencia en la didáctica de la enseñanza de una segunda lengua, en este caso, el inglés

Antes lo expuesto, y considerando que esta investigación es innovadora, ya que promueve la producción del texto en inglés mediante el aprendizaje cooperativo, se decidió abordar el tema. El objetivo del presente estudio fue determinar la influencia del Aprendizaje cooperativo en la producción de textos inglés en estudiantes del $1^{\circ}$ de secundaria JEC "Julio C. Tello", San Alejandro, Irazola.

\section{METODOLOGÍA}

Según Zunini y Cardoso (2005), el tipo de estudio fue experimental, porque se aplicó un estímulo a una de las variables con el fin de conseguir una modificación positiva en su comportamiento, ejerciendo control sobre las variables y sobre los sujetos de investigación.

a Jossara Evellyn Fernández-Blanco, b José Smith Landeo-Cabezudo y c Elizabeth Pacheco-Dávila Articulo Protegido por Licencia Creative Commons: BY-NC-ND / Protected by Creative Commons: BY-NC-ND. Sendas es una revista de acceso abierto / Sendas is an Open Access Journal. 
En la metodología de análisis y presentación de datos se recolectaron, elaboraron y procesaron en cuadros de distribución de frecuencias con sus gráficos respectivos.

La población corresponde a 185 estudiantes del VI ciclo del $1^{\circ}$ grado de secundaria de la Institución Educativa JEC "Julio C. Tello", San Alejandro, Irazola. Se trata de una muestra discrecional que quedó conformada por 60 estudiantes.

Respecto a la variable Producción de textos en inglés, se midió a través de dos dimensiones: i) Adecúa el texto a la situación comunicativa; ii) Organiza y desarrolla las ideas de forma coherente y cohesionada. La primera dimensión contiene cuatro indicadores: Propósito comunicativo, Destinatario, Tipo de texto, Género. La segunda dimensión contiene tres indicadores: Ordenación lógica de las ideas, Complementa sus ideas, Utiliza un vocabulario pertinente.

Considerando lo sostenido por Chiroque (2004), la técnica e instrumento que se empleó en el presente estudio fueron la Técnicas Análisis documental, que permitió determinar un instrumento de recolección de información directa sobre la producción de textos en inglés.

Mientras que el instrumento fue la Guía de análisis documental, que permitió recoger información de manera directa de las fuentes de información, a través de la evaluación en los indicadores previamente planteados, siendo esta prueba sujeta de validación por juicio de expertos y para la confiabilidad la prueba de Alfa de Cronbach.

La metodología de análisis y presentación de datos fueron la Recolección de datos y elaboración de la base de datos a través de los instrumentos y procesamiento de éstos en cuadros de distribución de frecuencias y sus gráficos respectivos. En la Prueba de hipótesis se empleó la t de student para muestras independientes.

\section{RESULTADOS}

Las respuestas a las preguntas planteadas se realizaron a partir de un análisis de los resultados que a continuación se exponen, referentes a la relación entre dos variables:

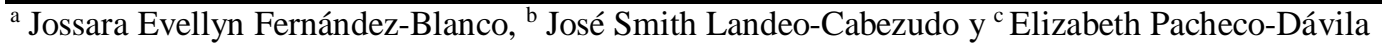
Articulo Protegido por Licencia Creative Commons: BY-NC-ND / Protected by Creative Commons: BY-NC-ND. Sendas es una revista de acceso abierto / Sendas is an Open Access Journal. 
Aprendizaje cooperativo y la producción de textos inglés, con el fin de analizar la influencia que ejerce la variable independiente sobre la variable dependiente.

\section{Tabla 01. Valor y criterio}

\begin{tabular}{|c|c|c|c|c|c|c|c|c|c|}
\hline \multirow{3}{*}{\multicolumn{2}{|c|}{$\begin{array}{l}\text { Producción } \\
\text { textos en inglés }\end{array}$}} & \multicolumn{4}{|c|}{ Grupo de Control } & \multicolumn{4}{|c|}{ Grupo Experimental } \\
\hline & & \multicolumn{2}{|c|}{ Pre Test } & \multicolumn{2}{|c|}{ Post Test } & \multicolumn{2}{|c|}{ Pre Test } & \multicolumn{2}{|c|}{ Post Test } \\
\hline & & \multirow{2}{*}{$\begin{array}{l}\mathrm{fi} \\
11\end{array}$} & \multirow{2}{*}{\begin{tabular}{|l|} 
hi $\%$ \\
$37 \%$
\end{tabular}} & \multirow{2}{*}{$\begin{array}{l}\text { fi } \\
8\end{array}$} & \multirow{2}{*}{$\frac{\text { hi \% }}{27 \%}$} & \multirow{2}{*}{$\begin{array}{l}\mathrm{Fi} \\
13\end{array}$} & \multirow{2}{*}{$\begin{array}{l}\text { hi } \% \\
43 \%\end{array}$} & \multirow{2}{*}{$\begin{array}{l}\text { fi } \\
2\end{array}$} & \multirow{2}{*}{$\begin{array}{l}\text { hi } \% \\
7 \%\end{array}$} \\
\hline Inicio & \begin{tabular}{|l}
01 \\
$10]$ \\
\end{tabular} & & & & & & & & \\
\hline Proceso & $\begin{array}{l}{[11} \\
13]\end{array}$ & 13 & $43 \%$ & 16 & $53 \%$ & 10 & $33 \%$ & 5 & $17 \%$ \\
\hline Esperado & $\begin{array}{l}{[14} \\
17]\end{array}$ & 6 & $20 \%$ & 6 & $20 \%$ & 7 & $23 \%$ & 15 & $50 \%$ \\
\hline Destacado & $\begin{array}{l}{[18} \\
20]\end{array}$ & 0 & $0 \%$ & 0 & $0 \%$ & 0 & $0 \%$ & 8 & $27 \%$ \\
\hline \multicolumn{2}{|l|}{ Total } & 30 & $100 \%$ & 30 & $100 \%$ & 30 & $100 \%$ & 30 & $100 \%$ \\
\hline \multicolumn{10}{|c|}{ Estadígrafos } \\
\hline \multicolumn{3}{|c|}{ Media Aritmética } & 11.20 & \multicolumn{2}{|c|}{11.87} & \multicolumn{2}{|l|}{11} & \multicolumn{2}{|c|}{14.80} \\
\hline \multicolumn{3}{|l|}{ Mediana } & 12 & \multicolumn{2}{|l|}{12} & \multicolumn{2}{|l|}{11} & \multicolumn{2}{|l|}{14} \\
\hline \multicolumn{3}{|c|}{ Desviación Estándar } & 2.483 & \multicolumn{2}{|c|}{2.063} & \multicolumn{2}{|c|}{3.0708} & \multicolumn{2}{|c|}{2.524} \\
\hline
\end{tabular}

Fuente: Base de datos

Figura2: Producción de textos en ingles

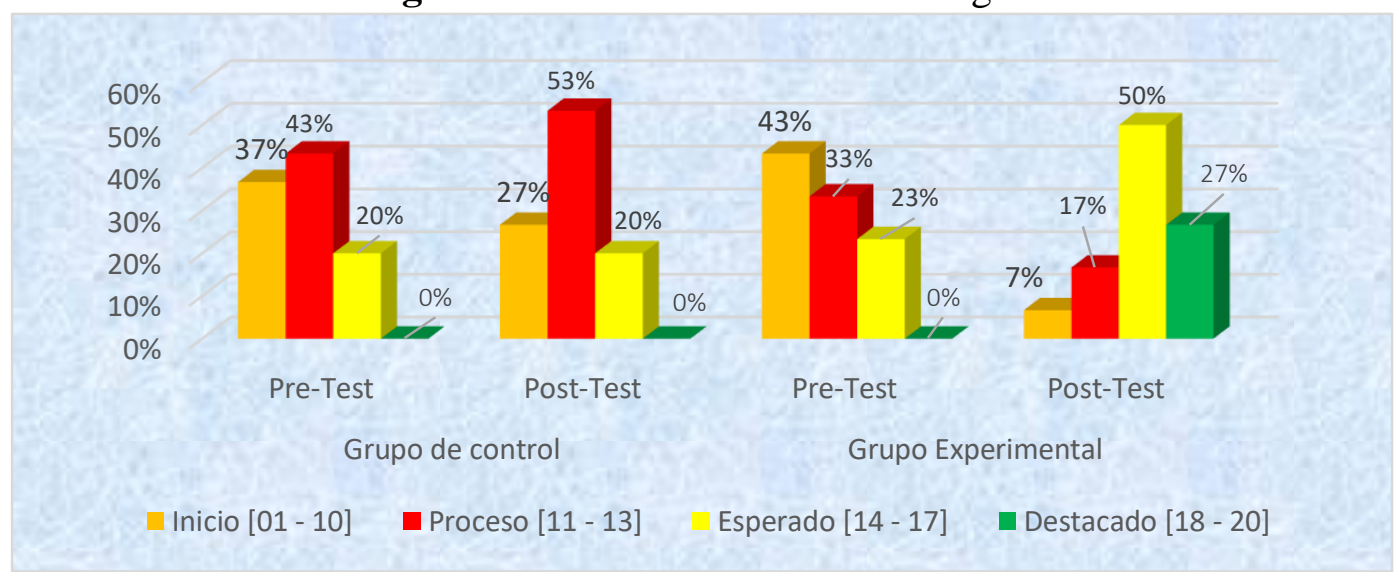

Producción de textos en inglés

En base a la tabla 1 y figura 2, se evidencia que el post test del grupo control tiene una media aritmética de 11.87, con una mediana de 12 y una desviación estándar de 2.063;

a Jossara Evellyn Fernández-Blanco, ${ }^{\mathrm{b}}$ José Smith Landeo-Cabezudo y ${ }^{\mathrm{c}}$ Elizabeth Pacheco-Dávila Articulo Protegido por Licencia Creative Commons: BY-NC-ND / Protected by Creative Commons: BY-NC-ND. Sendas es una revista de acceso abierto / Sendas is an Open Access Journal. 
además, con un $27 \%$ se encuentra en nivel inicio, el 53\% en proceso y un 20\% en esperado. Mientras que en el grupo experimental el post Test la media aritmética es de 14.80 con una mediana de 14 y una desviación estándar de 2.524; además, con un $7 \%$ se encuentra en nivel inicio, el $17 \%$ en proceso, un 50\% en esperado y un $27 \%$ en el nivel destacado. Esto quiere decir que el Aprendizaje cooperativo influye en la producción de textos en inglés.

\section{Tabla 2: Adecúa el texto a la situación comunicativo}

\begin{tabular}{|c|c|c|c|c|c|c|c|c|c|}
\hline \multirow{3}{*}{\multicolumn{2}{|c|}{$\begin{array}{l}\text { Adecúa el texto a la } \\
\text { situación } \\
\text { comunicativa }\end{array}$}} & \multicolumn{4}{|c|}{ Grupo de Control } & \multicolumn{4}{|c|}{ Grupo Experimental } \\
\hline & & \multicolumn{2}{|c|}{ Pre Test } & \multicolumn{2}{|c|}{ Post Test } & \multicolumn{2}{|c|}{ Pre Test } & \multicolumn{2}{|c|}{ Post Test } \\
\hline & & fi & hi $\%$ & fi & hi $\%$ & fi & hi \% & fi & hi \% \\
\hline Inicio & {$[01-10]$} & 14 & $47 \%$ & 12 & $40 \%$ & 15 & $50 \%$ & 4 & $13 \%$ \\
\hline Proceso & {$[11-13]$} & 3 & $10 \%$ & 7 & $23 \%$ & 5 & $17 \%$ & 3 & $10 \%$ \\
\hline Esperado & [14 - 17] & 13 & $43 \%$ & 11 & $37 \%$ & 10 & $33 \%$ & 19 & $63 \%$ \\
\hline Destacado & {$[18-20]$} & 0 & $0 \%$ & 0 & $0 \%$ & 0 & $0 \%$ & 4 & $13 \%$ \\
\hline Total & & 30 & $100 \%$ & 30 & $100 \%$ & 30 & $100 \%$ & 30 & $100 \%$ \\
\hline \multicolumn{10}{|c|}{ Estadígrafos } \\
\hline \multicolumn{3}{|c|}{ Media Aritmética } & 11.73 & \multicolumn{2}{|c|}{11.93} & \multicolumn{2}{|l|}{11} & \multicolumn{2}{|c|}{14.53} \\
\hline \multicolumn{3}{|l|}{ Mediana } & 12 & \multicolumn{2}{|l|}{12} & \multicolumn{2}{|l|}{11} & \multicolumn{2}{|l|}{14} \\
\hline \multicolumn{3}{|c|}{ Desviación Estándar } & 2.864 & \multicolumn{2}{|c|}{2.377} & \multicolumn{2}{|c|}{3.2695} & \multicolumn{2}{|c|}{2.874} \\
\hline
\end{tabular}

Fuente: Base de datos

Figura 3: Adecua el texto a la situación comunicativo

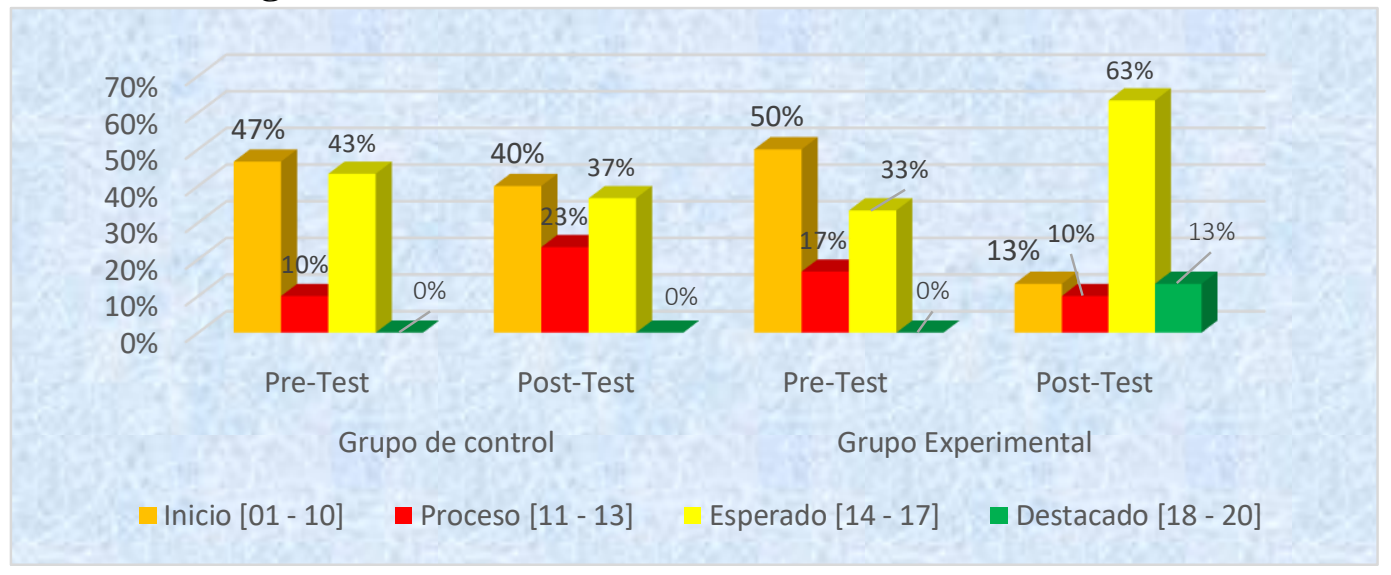

Fuente: Tabla 2

En base a la tabla 2 y figura 3, se evidencia que el post test del grupo control tiene la media aritmética de 11.93, con una mediana de 12 y una desviación estándar de 2.377;

a Jossara Evellyn Fernández-Blanco, ${ }^{\mathrm{b}}$ José Smith Landeo-Cabezudo y ${ }^{\mathrm{c}}$ Elizabeth Pacheco-Dávila Articulo Protegido por Licencia Creative Commons: BY-NC-ND / Protected by Creative Commons: BY-NC-ND. Sendas es una revista de acceso abierto / Sendas is an Open Access Journal. 
además, con un $40 \%$ se encuentra en nivel inicio, el $23 \%$ en proceso y un $37 \%$ en esperado. Mientras que el grupo experimental del post Test tiene una media aritmética de 14.53, con una mediana de 14 y una desviación estándar de 2.874; además, con un $13 \%$ se encuentra en nivel inicio, el $10 \%$ en proceso, un $63 \%$ en esperado y un $13 \%$ en el nivel destacado. Esto quiere decir que el Aprendizaje cooperativo influye en la producción de textos en inglés en la dimensión Adecúa el texto a la situación comunicativa.

Tabla 3: Organiza y desarrolla las ideas de forma coherente y cohesionada

\begin{tabular}{|c|c|c|c|c|c|c|c|c|c|}
\hline \multirow{3}{*}{\multicolumn{2}{|c|}{$\begin{array}{l}\text { Organiza } \\
\text { desarrolla las ideas } \\
\text { de forma coherente } \\
\text { y cohesionada }\end{array}$}} & \multicolumn{4}{|c|}{ Grupo de Control } & \multicolumn{4}{|c|}{ Grupo Experimental } \\
\hline & & \multicolumn{2}{|c|}{ Pre Test } & \multicolumn{2}{|c|}{ Post Test } & \multicolumn{2}{|c|}{ Pre Test } & \multicolumn{2}{|c|}{ Post Test } \\
\hline & & fi & hi \% & fi & hi $\%$ & fi & hi \% & fi & hi \% \\
\hline Inicio & [01 - 10] & 17 & $57 \%$ & 12 & $40 \%$ & 18 & $60 \%$ & 2 & $7 \%$ \\
\hline Proceso & {$[11-13]$} & 2 & $7 \%$ & 6 & $20 \%$ & 1 & $3 \%$ & 2 & $7 \%$ \\
\hline Esperado & [14 - 17] & 11 & $37 \%$ & 12 & $40 \%$ & 11 & $37 \%$ & 20 & $67 \%$ \\
\hline Destacado & [18 - 20] & 0 & $0 \%$ & 0 & $0 \%$ & 0 & $0 \%$ & 6 & $20 \%$ \\
\hline \multicolumn{2}{|l|}{ Total } & 30 & $100 \%$ & 30 & $100 \%$ & 30 & $100 \%$ & 30 & $100 \%$ \\
\hline \multicolumn{10}{|c|}{ Estadígrafos } \\
\hline \multicolumn{3}{|c|}{ Media Aritmética } & 10.67 & \multicolumn{2}{|c|}{11.80} & \multicolumn{2}{|l|}{11} & \multicolumn{2}{|c|}{15.07} \\
\hline \multicolumn{3}{|l|}{ Mediana } & 10 & \multicolumn{2}{|l|}{12} & \multicolumn{2}{|l|}{10} & \multicolumn{2}{|c|}{14} \\
\hline \multicolumn{3}{|c|}{ Desviación Estándar } & 3.457 & \multicolumn{2}{|c|}{2.644} & \multicolumn{2}{|c|}{3.3829} & \multicolumn{2}{|c|}{2.864} \\
\hline
\end{tabular}

Fuente: Tabla 3

Figura 3: Organiza y desarrolla las ideas de forma coherente y cohesionada

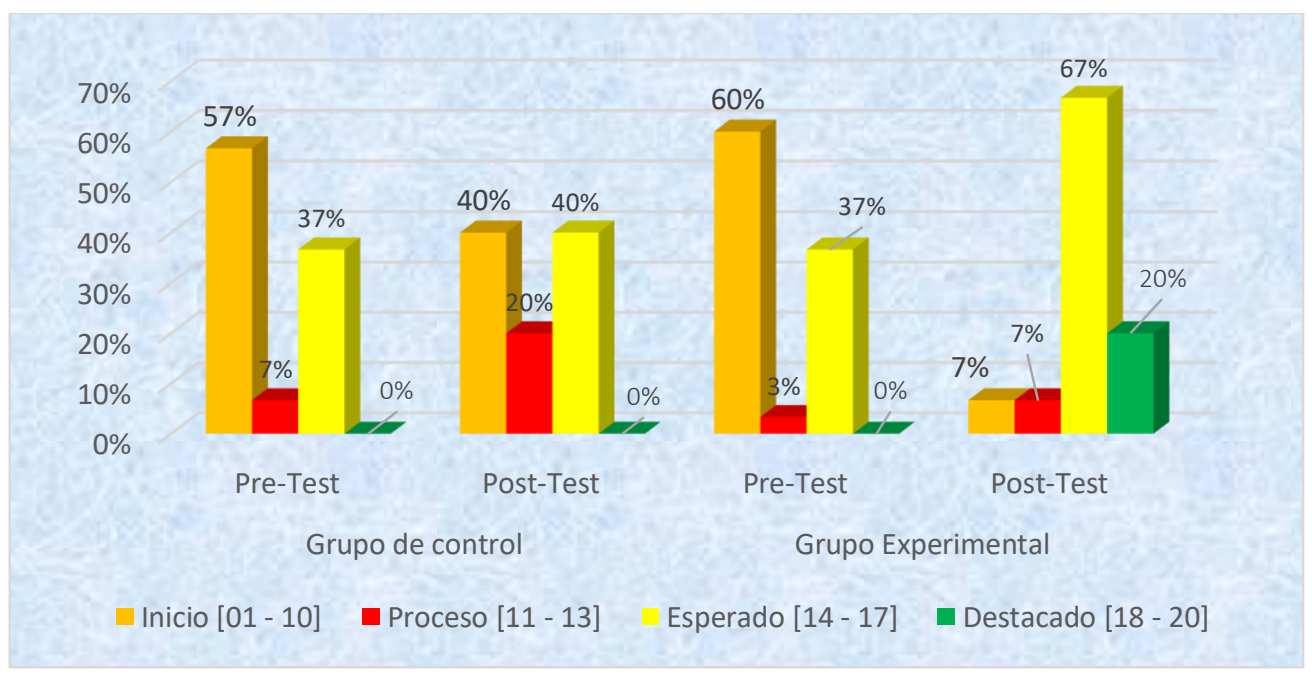

Fuente: Tabla 3

a Jossara Evellyn Fernández-Blanco, ${ }^{\mathrm{b}}$ José Smith Landeo-Cabezudo y ${ }^{\mathrm{c}}$ Elizabeth Pacheco-Dávila Articulo Protegido por Licencia Creative Commons: BY-NC-ND / Protected by Creative Commons: BY-NC-ND. Sendas es una revista de acceso abierto / Sendas is an Open Access Journal. 
En base a la tabla 3 y figura 4, se evidencia que el post test del grupo control tiene la media aritmética de 11.80, con una mediana de 12 y una desviación estándar de 2.644; Además, con un $40 \%$ se encuentra en nivel inicio, el $20 \%$ en proceso y un $40 \%$ en esperado. Mientras que, en el grupo experimental, el post Test tiene una media aritmética de 15.07, con una mediana de 14 y una desviación estándar de 2.864; además, con un $7 \%$ se encuentra en nivel inicio, el $7 \%$ en proceso, un $67 \%$ en esperado y un $20 \%$ en el nivel destacado. Esto quiere decir que el Aprendizaje cooperativo influye en la producción de textos en inglés en la dimensión Organiza y desarrolla las ideas de forma coherente y cohesionada.

\section{DISCUSIÓN}

Aprendizaje cooperativo proviene de dos términos: i) aprendizaje, según la RAE, en una de sus acepciones, significa "Acción y efecto de aprender algún arte u otra cosa, y ii) cooperativo, que proviene del latín cooperativus, y en una de sus acepciones significa "Que coopera o puede cooperar algo". En el presente trabajo se asumió que el aprendizaje cooperativo es el que se da en una situación de interacción cooperativa entre estudiantes en el medio educativo.

Producción de textos implica un proceso reflexivo porque supone la adecuación y organización de los textos considerando los contextos y el propósito comunicativo, así como la revisión permanente de lo escrito con la finalidad de mejorarlo. A decir de Inga (2008), producción de textos significa:

Escribir es producir mensajes a través de diversos tipos de textos, en función de una necesidad y de un determinado destinatario. Producir un texto no es transcribir o elaborar frases $u$ oraciones sueltas, es construir una unidad significativa completa sea breve. (p. 91)

El aprendizaje cooperativo es una estrategia metodológica a la que se recurre ante la dificultad de aprendizaje que afrontan los estudiantes de la Institución Educativa JEC “Julio C. Tello" en la producción de textos en inglés.

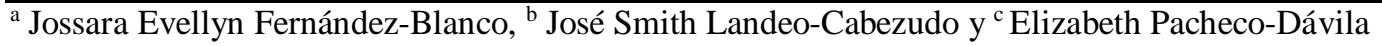
Articulo Protegido por Licencia Creative Commons: BY-NC-ND / Protected by Creative Commons: BY-NC-ND. Sendas es una revista de acceso abierto / Sendas is an Open Access Journal. 
Después de probar la hipótesis que sostiene el presente trabajo se concluyó que existe influencia significativa del Aprendizaje cooperativo sobre la Producción de textos en inglés, en estudiantes del $1^{\circ}$ de secundaria JEC "Julio C. Tello", San Alejandro, Irazola. Este resultado guarda relación con lo encontrado por De León (2013), en su tesis "El aprendizaje cooperativo como estrategia para el aprendizaje del idioma inglés", menciona que, al analizar los datos, se concluyó que la implementación de la metodología de aprendizaje cooperativo en el curso de inglés permitió un resultado positivo en el grupo experimental, más no significativo en términos estadísticos.

Esta afirmación coincide con lo que plantea. Pujolás, (2009), ya que resalta los aportes valiosos del aprendizaje cooperativo en otros aspectos educativos

Pues bien, todos estos procesos se dan más fácilmente en el aula si las tareas académicas comunes se realizan en régimen de interacción cooperativa, en lugar de individualista o competitiva. Una estructura de aprendizaje cooperativa permite fomentar interacciones positivas entre alumnos y entre estos y el profesor, por lo que se convierte en una estrategia instruccional de primer orden para facilitar el trabajo con un grupo heterogéneo, incluso con alumnos con necesidades educativas especiales en situaciones de integración escolar. (p. 13)

Como se puede ver, el valioso aporte del aprendizaje cooperativo va más allá del aprendizaje significativo y producción de texto, pues también desarrolla las habilidades sociales y comunicacionales de una lengua extranjera.

Estos hallazgos están respaldados por Stern, (1983), citado por Torres (2017), quien afirma que la enseñanza a través de las estrategias didácticas de una segunda lengua es un fenómeno bastante complejo y multidimensional que está supeditado a la interrelación de diferentes variables, así como a la importancia de la formación del profesorado, quienes deben tener muy en cuenta las inteligencias múltiples que poseen los estudiantes. Asimismo, dar las debidas recomendaciones a nivel de colegios e institucional.

a Jossara Evellyn Fernández-Blanco, b José Smith Landeo-Cabezudo y c Elizabeth Pacheco-Dávila Articulo Protegido por Licencia Creative Commons: BY-NC-ND / Protected by Creative Commons: BY-NC-ND. Sendas es una revista de acceso abierto / Sendas is an Open Access Journal. 
Asimismo, se demostró que existe influencia significativa del Aprendizaje cooperativo en la dimensión Adecúa el texto a la situación comunicativa en inglés en estudiantes del $1^{\circ}$ de secundaria. Estos resultados están respaldados por el hallazgo de Gutiérrez (2015). En su tesis concluyó que la aplicación del programa de la estrategia de aprendizaje cooperativo influye significativamente en la mejora de la producción de textos. Además, afirma también que el aprendizaje cooperativo es una innovación pedagógica eficaz que propicia el desarrollo de capacidades creativas. Estos hallazgos se ven respaldados por lo que afirma, Veliz Almeida (2009), sostiene que el aprendizaje cooperativo es un tipo de aprendizaje en el qué mediante la utilización de métodos, técnicas, procedimientos y formas para contextualizar el aprendizaje en los equipos de los alumnos, se procura establecer relaciones de cooperación para realizar el aprendizaje.

Así, al demostrar la hipótesis, concluimos que existe influencia significativa del Aprendizaje cooperativo en la dimensión Organiza y desarrolla las ideas de forma coherente y cohesionada en inglés en estudiantes del $1^{\circ}$ de secundaria. El texto en inglés cumplió una serie de reglas, relaciones semánticas y gramaticales, entre sus elementos constitutivos para que el lector decodifique el mensaje. En la coherencia las oraciones que guardan relación en torno a un asunto, tiene progresión y lógica entre sus enunciados y contenido global. Mientras que en la cohesión las ideas del texto fueron unidos adecuadamente. Según Halliday y Hasan (1976) la cohesión es una relación semántica entre oraciones a través de procedimientos gramaticales, cohesión léxica y conectores.

Ello se ve respaldado por lo que encontró Ojeda y Reyes (2006), en sus tesis; concluyen que las estrategias de aprendizaje cooperativo son una alternativa y uno de los caminos o medios más eficaces para alcanzar aprendizajes óptimos y significativos; además permite que los alumnos se integren y aprendan en equipo en la construcción de nuevas capacidades, conocimientos y comportamientos escolares muy diferentes a los estilos de aprendizaje individual. Al respecto, La Prova (2017) sostiene lo siguiente:

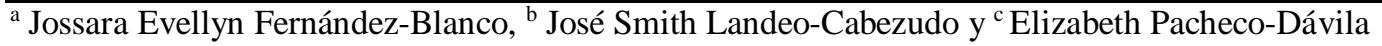
Articulo Protegido por Licencia Creative Commons: BY-NC-ND / Protected by Creative Commons: BY-NC-ND. Sendas es una revista de acceso abierto / Sendas is an Open Access Journal. 
En resumen, el Aprendizaje Cooperativo (AC) es un método de enseñanza/aprendizaje que actúa con los recursos del grupo, con el objetivo principal de mejorar el aprendizaje y las relaciones sociales. La premisa de fondo es que el grupo es un universo de recursos, no sólo de conocimientos sino también de competencias, por lo que la enseñanza/aprendizaje es un proceso no de “Transmisión” del profesor a los alumnos, sino de participación e intercambio entre todas las personas implicadas. Como subraya Gentile (2004), los alumnos se convierten en protagonistas activos de su propio aprendizaje ya que están implicados en actividades que les "hacen encajar" en un juego de interdependencia que no les permite sustraerse al trabajo común. (p.10)

Estos hallazgos están respaldados por Díaz (1999), quien afirma que el aprendizaje debe garantizar que sea cooperativo de la siguiente manera: La igualdad de organización y desarrollo de cada individuo en el proceso de aprendizaje; la realización de experiencias mutuas en el aula entre los diferentes compañeros de manera que haya una bidireccional en el proceso de enseñanza aprendizaje (unos aprenden de los otros) y la distribución de responsabilidades y el intercambio de roles. Por otra parte, Rutherford et al (1998), Arias, Cárdenas y Estupiñan (2005) complementa así: "El aprendizaje cooperativo se basa en la estructura organizacional de los grupos y más específicamente en el poder motivacional de las relaciones con otras personas" (p.16).

De lo expuesto, se puede inferir que no basta con organizar el aprendizaje cooperativo también hay que garantizar que el docente y estudiante conozcan su estructura para no fracasar en el intento.

\section{CONCLUSIONES}

1. En base a la tabla 1 y figura 2 se evidencia que en el post test del grupo control, tiene una media aritmética de 11.87 con una mediana de 12 y una desviación estándar de 2.063, además, con un $27 \%$ se encuentra en nivel inicio, el $53 \%$ en proceso y un $20 \%$

a Jossara Evellyn Fernández-Blanco, b José Smith Landeo-Cabezudo y c Elizabeth Pacheco-Dávila Articulo Protegido por Licencia Creative Commons: BY-NC-ND / Protected by Creative Commons: BY-NC-ND. Sendas es una revista de acceso abierto / Sendas is an Open Access Journal. 
en esperado. Mientras que en el grupo experimental el post Test, se evidencia una media aritmética de 14.80 con una mediana de 14 y una desviación estándar de 2.524, además, un $7 \%$ se encuentra en nivel inicio, el $17 \%$ en proceso un 50\% en esperado y un $27 \%$ en el nivel destacado.

La influencia del aprendizaje cooperativo en la producción de textos en inglés. En base al $\mathrm{P}-$ valor $=0.000$, que es menor que el $\propto=0.025$. Se concluye que existe influencia significativa del Aprendizaje cooperativo en la producción de textos inglés en estudiantes del $1^{\circ}$ de secundaria JEC "Julio C. Tello, San Alejandro, Irazola, 2018

2. En base a la tabla 2 y figura 3, se evidencia que en el post test del grupo control, tiene la media aritmética de 11.93 con una mediana de 12 y una desviación estándar de 2.377, además, con un $40 \%$ se encuentra en nivel inicio, el $23 \%$ en proceso y un $37 \%$ en esperado. Mientras que en el grupo experimental el post Test, tiene una media aritmética es de 14.53 con una mediana de 14 y una desviación estándar de 2.874, además, con un $13 \%$ se encuentra en nivel inicio, el $10 \%$ en proceso un $63 \%$ en esperado y un $13 \%$ en el nivel destacado, evidenciándose, la influencia del aprendizaje cooperativo en la producción de textos en inglés, en la dimensión, adecúa el texto a la situación comunicativa.

Además, en función al $\mathrm{P}-\mathrm{valor}=0.000$, que es menor que el $\propto=0.025$. Por lo que concluimos que existe influencia significativa del aprendizaje cooperativo en la dimensión Adecúa el texto a la situación comunicativa en inglés en estudiantes del $1^{\circ}$ de secundaria.

3. En base a la tabla 3 y figura 4 , se evidencia que en el post test del grupo control, tiene la media aritmética de 11.80 con una mediana de 12 y una desviación estándar de 2.644, además, con un $40 \%$ se encuentra en nivel inicio, el $20 \%$ en proceso y un $40 \%$ en esperado. Mientras que en el grupo experimental el post Test, tiene una media aritmética es de 15.07 con una mediana de 14 y una desviación estándar de 2.864, además, con un $7 \%$ se encuentra en nivel inicio, el $7 \%$ en proceso un $67 \%$ en esperado y un $20 \%$ en el nivel destacado, evidenciándose, la influencia del aprendizaje

a Jossara Evellyn Fernández-Blanco, ${ }^{\mathrm{b}}$ José Smith Landeo-Cabezudo y ${ }^{\mathrm{c}}$ Elizabeth Pacheco-Dávila Articulo Protegido por Licencia Creative Commons: BY-NC-ND / Protected by Creative Commons: BY-NC-ND. Sendas es una revista de acceso abierto / Sendas is an Open Access Journal. 
cooperativo en la producción de textos en inglés, en la dimensión, Organiza y desarrolla las ideas de forma coherente y cohesionada.

Además, en función al $\mathrm{P}-\mathrm{valor}=0.000$, que es menor que el $\propto=0.025$. Concluimos que existe influencia significativa del aprendizaje cooperativo en la dimensión Organiza y desarrolla las ideas de forma coherente y cohesionada en inglés en estudiantes del $1^{\circ}$ de secundaria.

\section{REFERENCIAS}

ANDINA (2016). Perú tiene nivel bajo el dominio de inglés, según ranking mundial. En: andina.pe > agencia > noticia-peru-tiene-nivel-bajo-dominio-ingles-se...

Arias, J., Cárdenas, C. y Estupiñan, F. (2005). Aprendizaje cooperativo. Segunda Edición. Colombia: Universidad Pedagógica Nacional.

De León (2013). "El aprendizaje cooperativo como estrategia para el aprendizaje del idioma inglés” Tesis. Quetzaltenango, Guatemala: Universidad Rafael Saldívar.

Duran, D. y Vidal, V. (2004). Tutoría entre iguales: de la teoría a la práctica. Un método de aprendizaje cooperativo para la diversidad en secundaria. España: GRAO.

Gutierrez, S. (2015). "Estrategias cooperativo en la producción de textos ingresantes a la institución Educativa emblemática José María Arguedas de Chincheros Apurímac”, Tesis. Lima: UNE “Enrique Guzmán y Valle”.

Inga, M. (2008). Taller de Comunicación Integral. CPEI UNMSM: Lima

Jara, N., Constanza, F y Romero, F. (2016). "El aprendizaje cooperativo mejora el aprendizaje del inglés como lengua extranjera en estudiantes de ler año medio de un colegio particular subvencionado de la comuna de Talcahuano". Tesis de Licenciatura. Colombia: Universidad Católica de la Santísima Concepción.

La Prova, A. (2017). La práctica del Aprendizaje Cooperativo: Propuestas operativas para el grupo - clase. España: NARCEA S.A. DE EDICIONES.

Pujolás, P. (2009). 9 ideas clave. El aprendizaje cooperativo. España: Editorial GRAO.

a Jossara Evellyn Fernández-Blanco, b José Smith Landeo-Cabezudo y c Elizabeth Pacheco-Dávila Articulo Protegido por Licencia Creative Commons: BY-NC-ND / Protected by Creative Commons: BY-NC-ND. Sendas es una revista de acceso abierto / Sendas is an Open Access Journal. 
Unesco (2014). Aportes para la enseñanza de la escritura y comprensión de lectura. Consultado en: http://unesdoc.unesco.org/images/0024/0024s.pdf

Zunini y Cardoso (2005). Taller de investigación II. Lambayeque: Universidad Nacional Pedro Ruiz Gallo. En: https://es.slideshare.net/BrandonTorres20/3-factores-queintervienen-en-el-aprendizaje-y-adquisicion-de-la-segunda-lengua

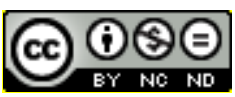

Aprendizaje cooperativo en la producción de textos en inglés en estudiantes de secundaria JEC (Jossara Evellyn Fernández-Blanco) Por Revista Kolpa se encuentra bajo una Licencia Creative Commons-No Comercial-Sin Derivadas 3.0 Uported.

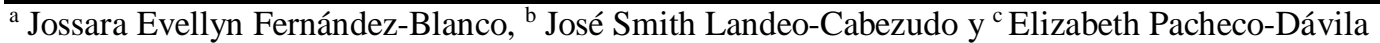
Articulo Protegido por Licencia Creative Commons: BY-NC-ND / Protected by Creative Commons: BY-NC-ND. Sendas es una revista de acceso abierto / Sendas is an Open Access Journal. 